\title{
Effect of the initial temperature on the water-reducing performance of polycarboxylate-based superplasticizers
}

\author{
Jinwen Wang ${ }^{1, \text { a }}$, Guangjun Zheng ${ }^{1, \mathrm{~b}}$, Minghua Yuan ${ }^{1}$, Chong $\mathrm{Sun}^{1}, \mathrm{Yao} \mathrm{Bi}^{1, \mathrm{c}}$ \\ ${ }^{1}$ Department of Technology Center, China State Construction Ready Mixed Concrete CO., LTD, \\ Wuhan, 430074, China \\ a email: wangjinwench@163.com ${ }^{b}$ email: jaylia08@hotmail.com ${ }^{\mathrm{c}}$ email: freddiebee@163.com
}

Keywords: Superplasticizers, Water-reducing Performance, Initial temperature, Fluidity

\begin{abstract}
In order to study the effect of the temperature of reaction system on the properties of polycarboxylate-based superplasticizers (PC) and on the cement workability, the PC are synthesized in different initiatory temperature from $10 \mathrm{C}$ to $40 \mathrm{C}$. Fourier transform infrared spectroscopy (FTIR), and gel permeation chromatography (GPC) are used for structural characterization. The experimental data show that the PCs synthesized at different initiatory temperature have similar functional groups but disparate molecular weight and its distribution. As a result, the PCs synthesized at the higher initiatory temperature of reaction system possess lower molecular weight and narrower distribution, resulting in higher initial fluidity but smaller fluidity retention capacity.
\end{abstract}

\section{Introduction}

Superplasticizers are nowadays becoming indispensable components in concrete to improve the workability of fresh concrete with less water-cement ratio by dispersing agglomerated hydrating cement particles present in the paste[1][2]. Different superplasticizers exhibit different adsorption behaviors and retardation effects, closely correlating with their specific molecular architecture. Among the sulfonated melamine formaldehyde (SMF) condensate, sulfonated naphthalene formaldehyde (SNF) condensate and acrylic polymer (AP), polycarboxylate superplasticizers with carboxylic groups and polyethylene oxide (PEO) side chains are considered as the third generation superplasticizers, due to their outstanding water reducing performance and potentially designable molecular structure. It is widely considered that the carboxylic groups act as anchors to adsorb on the surfaces of cement particles, and PEO side chains produce steric hindrance to prevent cement particles flocculating and hydrating[3].

Effective design of the molecular architecture will increase the adsorption efficiency of PCEs on particles, and also optimizes the steric hindrance; both of these factors are essential in achieving good plasticizing performance. Extensive researches have been carried out for better understanding and explaining the structure-property relationship of superplasticizers. Sayed N etc. [4] investigated the effects of superplasticizers with varied structures and found that PCs with higher molecular weight, lower side chain density and shorter side chains were favorable to a high adsorption amount on cement surface. J.Plank etc. [5] reported that not only the content of the carboxyl groups, but also their repartition along the polymer backbone would significantly affect the adsorption behaviors and the retardation effects of the carboxyl groups containing polymers.

Previous researches mainly concerned the effects of the various functional groups and the charge species of backbone in PCs on the adsorption, rheological and hydration behaviors of cement paste [6][7]. There is so far no study dedicated to the effects of initial synthesis temperature of reaction system on the adsorption and cement hydration. Since the temperature of reaction system affect not only the efficiency and rate of redox initiator systems but also the reactivity of active monomers, the chain structure and molecular weight distribution of the superplasticizers can undergone a corresponding change. This study aims at elucidating the correlations of initial synthesis temperature of PC superplasticizers with their adsorption behaviors and water reducing performance in cement pastes and to lay the experimental preclinical for the development of new superplasticizers with more efficient and robust performance. 


\section{Experimental}

\subsection{Raw materials}

Isopropyl oxy polyethylene glycol (TJ188, with Mw 2400) was supplied by Shanghai Taijie Chemical, China. Acrylic acid (AA), sodium methallyl sulfonate (SMS), thioglycolic acid (TGA), hydrogen peroxide $\left(\mathrm{H}_{2} \mathrm{O}_{2}\right)$, ascorbic acid $(\mathrm{Vc})$ and sodium hydroxide $(\mathrm{NaOH})$ are purchased from Sinopharm Chemical Reagent Co., Ltd.

The cement used in this study was an ordinary Portland cement (PO, 42.5R), supplied by Yadong cement plant, and the properties including chemical compositions density, specific surface and initial setting time are shown in Table 1.

Table 1 Chemical composition and physical properties of cement

\begin{tabular}{|c|c|c|c|c|c|c|c|c|c|c|}
\hline \multirow{2}{*}{ Materials } & \multicolumn{7}{|c|}{ Chemical compositions (\%) } & \multirow{2}{*}{$\begin{array}{l}\text { Specific } \\
\text { surface } \\
\left(\mathrm{m}^{2} / \mathrm{kg}\right)\end{array}$} & \multirow{2}{*}{$\begin{array}{l}\text { Density } \\
\left(\mathrm{kg} / \mathrm{m}^{3}\right)\end{array}$} & \multirow{2}{*}{$\begin{array}{l}\text { Initial } \\
\text { setting time } \\
\quad(\min )\end{array}$} \\
\hline & $\mathrm{SiO}_{2}$ & $\mathrm{Al}_{2} \mathrm{O}_{3}$ & $\mathrm{Fe}_{2} \mathrm{O}_{3}$ & $\mathrm{CaO}$ & $\mathrm{MgO}$ & $\mathrm{SO}_{3}$ & Loss & & & \\
\hline Cement & 21.7 & 8.01 & 3.64 & 59.3 & 2.32 & 2.08 & 2.18 & 338 & 3.02 & 185 \\
\hline
\end{tabular}

\subsection{Synthesis of PCs}

Four polymers (designated as PC-10, PC-20, PC-30 and PC-40) are synthesized at specific initial temperature from $10 \mathrm{C}$ to $40 \mathrm{C}$. TJ188 were dissolved in distilled water, when the temperature rises to specific initial temperature. $\mathrm{H}_{2} \mathrm{O}_{2}$ and AA aqueous solution, and TGA and Vc aqueous solution were added drop-wise into the reaction mixture separately. The round bottom flask was covered with rubber insulation cotton, which would keep the spread of the heat in reaction system. All the copolymerization reactions are carried out in an aqueous medium for 4 hours using hydrogen peroxide as an initiator and ascorbic acid as an activator, respectively, under a nitrogen gas atmosphere. Finally, the aqueous PCs solution was cooled to ambient and its $\mathrm{pH}$ was adjusted to 7 using 32 wt. \% aqueous $\mathrm{NaOH}$.

\subsection{Evaluation}

The FTIR spectrum of the disk was recorded with AVATAR-370 infrared spectroscopy (Thermo Nicolet Company, US) working at a resolution of $4 \mathrm{~cm}^{-1}$ and an accumulation of 32 scans.

The number average molecular weight $(\mathrm{Mn})$, weight average molecular weight $(\mathrm{Mw})$, and polydispersity index (PDI) of synthesized PCs were determined by gel permeation chromatography (GPC) using $0.1 \mathrm{~mol} / \mathrm{L}$ buffer solution of phosphoric acid as a mobile phase at a flow rate of 1.0 $\mathrm{mL} / \mathrm{min}$.

The fluidity of fresh cement paste with amide-PCE was tested according to the standard method GB/T8077-2000 described in the National Standards of People’s Republic of China. Generally, the cement pastes are prepared at a water to cement ratio (w/c) of 0.29 with the PC dosage of $0.15 \%$ against the mass of cement using an epicyclical mixer. The spread diameter of the cement cake is measured, which is used for measuring its fluidity. The cement paste is monitored over time to determine paste loss, and measurements are made every 30 min up to $150 \mathrm{~min}$.

\section{Results and discussion}

\subsection{The temperature variety curve of the reaction system}

Plots of the temperature rise change versus the reaction time are shown in Fig.1 for the polymerization reaction system with increasing initial temperature (from $10 \mathrm{C}$ to $40 \mathrm{C}$ ). These plots show differences in initial temperature of polymerization reaction that result in different temperature rise trend at a given time. The initial period is induction period, which decrease with the increase of initial temperature. That is, the higher the initial temperature of reaction system, the faster the rate of initiation, leading to obvious decrease in an induction period of polymerization. The second period is rapid polymerization reaction period leading to the immediately increasing temperature that occurs from the copolymerization of TJ188 and AA. The polymerization reaction 
period decreased with the increasing reaction temperature, which indicate it seem to increase significantly with the more highly initial temperature. The last period is reaction retention period, which is for raising the conversion ratio of monomers.

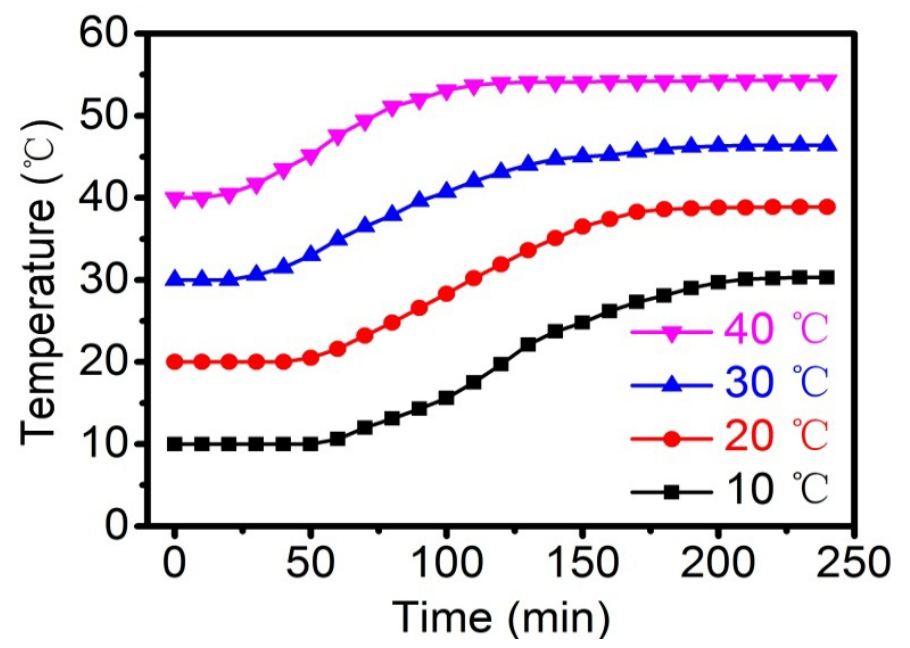

Fig.1 The temperature variety curve of the reaction system

\subsection{Characterization of synthesized PCs by the FT-IR spectrum}

As the FTIR spectrum has been proved to be a useful tool to effectively character the functional group of polymer[8], the infrared spectrum for the PCs synthesized at different initiatory temperature are given in Fig.2. The characteristic absorption peak of $C=C\left(1650 \mathrm{~cm}^{-1}\right)$ disappeared and the absorption peak intensity of carbon hydrogen bonds enhanced markedly, which provides a clear evidence of the completely polymerization of various monomers. The broad peak around 3455 $\mathrm{cm}^{-1}$ and the sharp peak at $2881 \mathrm{~cm}^{-1}$ are respectively assigned to the $\mathrm{O}-\mathrm{H}$ and $\mathrm{C}-\mathrm{H}$ stretching bands. The peak at $1726 \mathrm{~cm}^{-1}$ is assigned to the overlapping of $\mathrm{C}-\mathrm{O}$ in AA repeating units and the macro-monomer TJ188 units. Moreover, the characteristic band of macro-monomer TJ188 units also appears at $1132 \mathrm{~cm}^{-1}$ for the C-O-C asymmetric stretching. The band at $1468,1340 \mathrm{~cm}^{-1}$ arises from the $\mathrm{C}-\mathrm{H}$ stretching and bending vibration in the methylene group.

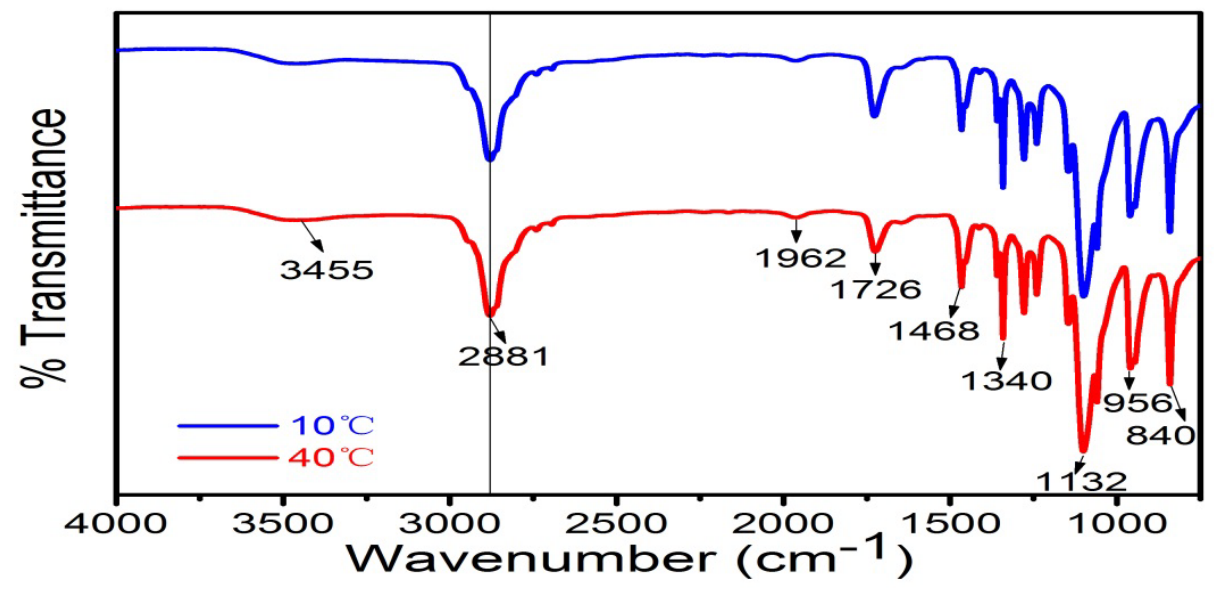

Fig.2 The infrared spectrum for the PCs synthesized at different initiatory temperature

According to the result of the FT-IR spectrum, it is obviously proved the functional groups have been successfully copolymerized to the molecular structure of PCs. However, the PCs synthesized at different initiatory temperature is not shown much difference in the whole spectrum, except for little varied intensity of peaks in some groups, which can easily be deduced those PCs have similar functional groups. 


\subsection{The GPC results of PCs synthesized at different initiatory temperature}

The GPC results of PCs synthesized at different initial temperatures are shown in Fig.3. It is found that the PCs possess a marked decreased peak height and increased peak width, as the initial temperatures increase. The polydispersity index (PDI) is obtained by dividing the weight average molecular weight $(\mathrm{Mw})$ by the number average molecular weight $(\mathrm{Mn})$. It describes the uniformity of a polymer with respect to molecular mass distribution. It can also be seen that polymer molecules with higher molecular weight elute earlier from the chromatography column. The initiator and monomers possess lowered reaction activity under inferior initial temperatures, which are rapid and dramatic in initiating the polymerization, resulting smaller molecular weight and wider distribution than higher initial temperatures.

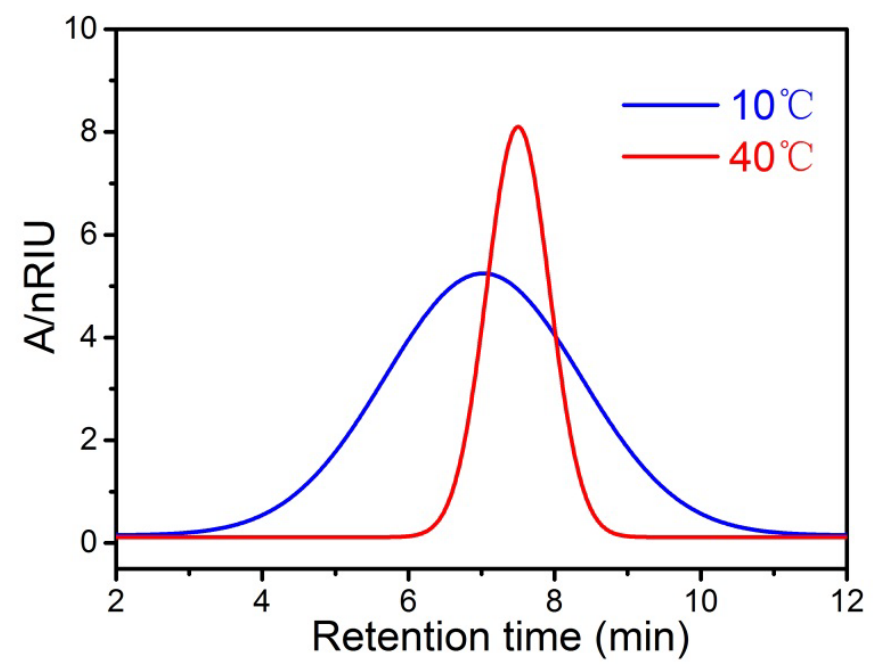

Fig.3 The GPC results of PCs synthesized at different initiatory temperature

\subsection{Effect of different PCs on dispersion of cement pastes}

In order to compare the performance of the synthesized PC co-polymers, the fluidity of the fresh cement pastes with the addition of various PCs synthesized at different initiatory temperature is measured as shown in Fig 4. The fluidity of cement paste is affected by the physical and chemical interactions between the cement and the superplasticizers [9]. The inclusion of superplasticizers, which are adsorbed onto the cement particles, prevents flocculation and water entrapment in the floccule, reducing the amount of water needed to obtain similar fluidity in the pastes. It is can be seen the initial fluidity of the cement pastes is increased with the initial temperature of reaction system, which is largely reduced from $235 \mathrm{~mm}$ to $325 \mathrm{~mm}$. However, the fluidity retention capacity of the cement pastes is shown an obviously contradictory trend.

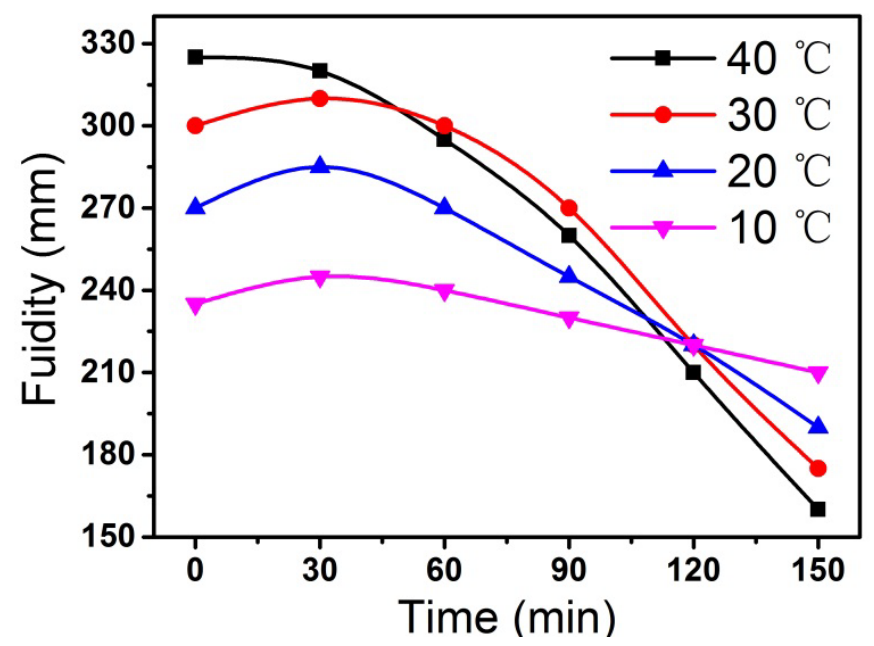

Fig.4 Effect of initial temperature and time elapsed on fluidity of cement pastes.

As the infrared spectrum drawing of PC-10 is similar to that of PC-40, it shows that PCs 
synthesized at different initial temperature has same chemical component and molecular configuration. Furthermore, despite of the same side chain lengths and ratios of side chain density to backbone charge, the addition of PCs show various initial fluidity and retention capacity of the cement pastes, which are attributed to different molecular weight and its distribution. However, it was noted that as the initial temperature decreased, the molecular weight distribution characterized by the polydispersity index (PDI) became relatively wide, which might be caused by relatively wide temperature range. Previous studies [10] on cement-water-superplasticizers systems have already demonstrated that the dispersion ability of PCs is partially related to molecular weight and its distribution. Therefore, it can be inferred that the relatively wide molecular weight distribution also played an important role in maintaining dispersion and slump loss behaviors of cement pastes.

\section{Conclusions}

In this study, PCs synthesized at different initiatory temperature were employed to investigate the influences of the initiatory temperature of reaction system on the water-reducing performance in cement pastes, which was shown that initiatory temperature greatly influences this property. The initiator and monomers possessed lowered reaction activity under inferior initial temperatures, resulting smaller molecular weight and wider distribution than higher initial temperatures. As a result, the initial fluidity of the cement pastes was increased with the initial temperature of reaction system, while the fluidity retention capacity of the cement pastes was shown an obviously contradictory trend.

\section{Reference}

[1] Alireza K, John L et al. Effect of molecular architecture of polycarboxylate ethers on plasticizing performance in alkali-activated slag paste [J]. J Mater Sci, 2014(49):2761-2772.

[2] Alone M, Palacios M, et al. Compatibility between polycarboxylate-based admixtures and blended-cement pastes [J]. Cement and Concrete Composites, 2013, (35):151-162.

[3] Ling J, Xiang K et al. Preparation of Amphoteric Polycarboxylate Superplasticizers and Their Performances in Cementations System [J]. Journal of Applied Polymer Science 2015, DOI: 10.1002/APP.41348.

[4] Sayed N, Latipa K, et al. Effects of Surfactants on the Properties of Mortar Containing Styrene/Methacrylate Superplasticizers [J].The Scientific World Journal, 2014(94):1-10.

[5] Lange A, Plank J et al. Influence of the HLB value of polycarboxylate superplasticizers on the flow behavior of mortar and concrete [J]. Cement and Concrete Research, 2014(60) 45-50.

[6] Janowska E. The effect of superplasticizers' chemical structure on their efficiency in cement pastes [J]. Construction and Building Materials, 2013(38):1204-1210.

[7] Xiao L, Ziming W et al. Preparation, Characterization and performances of powdered polycarboxylate superplasticizers with bulk polymerization [J]. Materials 2014(7):6169-6183.

[8] Yinwen , Chaolong Y. Study on dispersion, adsorption and flow retaining behaviors of cement mortars with TPEG-type polyether kind polycarboxylate superplasticizers [J].Construction and Building Materials,2014(64):324-332.

[9] Yinwen L, Huilong G et al. Synthesis of copolymers with cyclodextrin as pendants and its end group effect as superplasticizers [J]. Carbohydrate Polymers, 2014(102):278-287.

[10] Xiao L, Ziming W. Synthesis, characterization and performance of polycarboxylate superplasticizers with amide structure [J]. Colloids and Surfaces A, 2014(448):119-129. 